\title{
Research of MIMO-OFDM Model
}

\author{
Dong Qingwei ${ }^{1}$, Zhou Ling ${ }^{2}$, Liu Hui ${ }^{1}$, Wang Chunyu ${ }^{1}$ and Li Yaochun ${ }^{1}$ \\ ${ }^{1}$ Department of Aircraft Control, Aviation University of Air Force, Changchun, 130022, China. \\ ${ }^{2}$ East China Jiao Tong University, Nan Chang, 330013, China.
}

Keywords: MIMO,OFDM, Model,Multi-path, Fading.

\begin{abstract}
OFDM (Orthogonal Frequency Division Multiplex) technique is one of the promising trends of the new generation wireless telecommunication. It can be combined with MIMO (Multiple Input Multiple Output) technique. The MIMO-OFDM system can improve the performance and the capacity of the wireless telecommunication, suppress co-channel interference and multiple access interference, and achieve the diversity gain as resisting multi-path fading. The advantages and models of these two technologies are discussed according to their definitions. The advantage and model of MIMO-OFDM technology is discussed based on the complementary of these two technologies. The block diagram of MIMO-OFDM system is presented in the end.
\end{abstract}

\section{MIMO Model}

MIMO (Multiple Input Multiple Output) can be defined simply as following: In any wireless communication system, several antennas are employed in the transmitting and receiving terminal of the link. Its core thinking is the spatial and temporal signal processing. In other words, several antennas are employed to add the spatial dimension to the communication system[1]. Its aim is to realize multiple dimensions signal processing while the temporal dimension, which is adopted in the traditional communication system, is also used. The principle of MIMO technique is that the serial data are mapped to the parallel data, and are propagated[2]. In the receiving terminal, the parallel data are combined to the serial data, and the received signals are distinguished through the noncorrelation of the different antennas signal in the wireless channel. In order to assure the noncorrelation of the signals, the spacing of different antennas must be large. So the MIMO system is fitting to be used in the environment of multi-paths of serious scattering[3]. Even on the condition of Rayleigh fading of no straight path it can work better[4]. The MIMO technique can change the multi-paths effect factor of the traditional communication system to the advantaged factor for the communication performance, and it can increase the transmitting rate of the wireless communication system. This change need not increase the added signal bandwidth and the launching power. Furthermore the MIMO technique can resist the random fading effectively, and improve the performance of the wireless communication system greatly.

In principle, MIMO offers three different benefits, namely spatial diversity, beamforming gain, and spatial multiplexing. The stunning novelty of MIMO, however, was that channels could support parallel data streams by transmitting and receiving on orthogonal spatial channels (spatial multiplexing). The number of usefully multiplexed streams depends on the rank of the instantaneous channel matrix, which, in turn, depends on the spatial properties of the radio environment. The spatial multiplexing gain may reach in a sufficiently rich scattering environment. Spatial correlation - manifest as channel matrix rank reduction - will reduce this gain, as it will reduce diversity order.

The Fig. 1 is the block diagram of the MIMO system, which has $M$ transmit antennas and $N$ receive antennas. The signals at the transmit antenna elements is given as

$$
\mathbf{x}(t)=\left[x_{1}(t), x_{2}(t), \cdots, x_{M}(t)\right]^{T}
$$

Where $[\square]^{T}$ is the transposing of the vector or the matrix and $x_{i}(t)$ is the signal at ith transmit antenna element. 


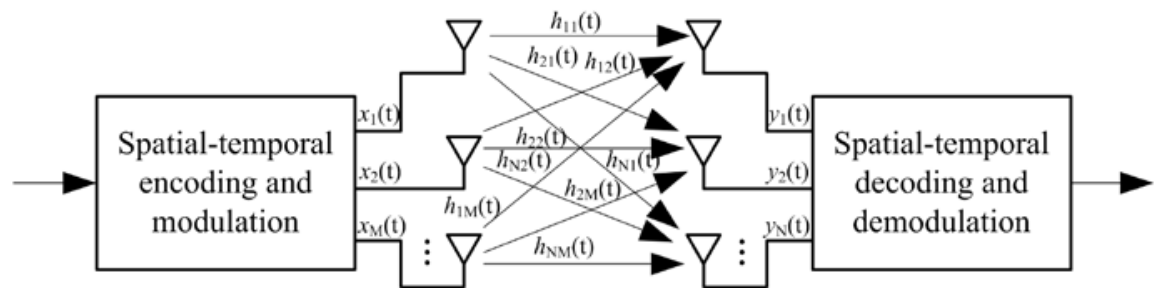

Fig.1: MIMO system model

The signals at the receive antenna elements is given as

$$
\mathbf{y}(t)=\left[y_{1}(t), y_{2}(t), \cdots, y_{N}(t)\right]^{T}
$$

Where $y_{j}(t)$ is the signal at the $j$ th receive antenna element.

The channel is assumed as the frequency flat fading channel. The sub-channels between antennas can be equivalent to the Rayleigh fading sub-channels.

The sub-channels of MIMO channel model can be represented respectively as

$$
h_{j, i}(\tau, t)=h_{j, i}(t) \delta\left(\tau-\tau_{0}\right)
$$

Where $i=1, \cdots, M ; j=1, \cdots, N$.

$\left|h_{j, i}(t)\right|$ follows the Rayleigh distribution.

So the MIMO channel matrix can be represented as

$$
\mathbf{H}=\left(h_{j, i}\right)_{N \times M}
$$

So the corresponding MIMO system can be represented as

$$
\mathbf{Y}=\mathbf{H X}+\mathbf{Z}
$$

Where $\mathbf{Z}$ is the Gaussian white noise matrix, whose mean is zero.

\section{OFDM Model}

OFDM (Orthogonal Frequency Division Multiplex) is the special technique of the special MCM (Multi-Carrier Modulation), and is the high rate propagation technique in the wireless communication environment[5]. Since the future wireless communication system is wideband, the frequency response of the wireless channel is usually non-flat[6]. In other words, it is the frequency selective fading channel. The OFDM technique divides the channel into many orthogonal subchannels in frequency domain. The short band modulation and propagation can be made on every sub-channel. So the co-inference between sub-channels can be decreased, and the spectrum utilizing rate can be increased in the mean while. Since the signal bandwidth of every sub-channel is less than the correlation bandwidth of the channel, the fading of every sub-channel is flat and the intersymbol interference can be decreased greatly.

OFDM is used extensively in broadband wired and wireless communication systems because it is an effective solution to inter symbol interference (ISI) caused by a dispersive channel. This becomes increasingly important as data rates increase to the point where, when conventional serial modulation schemes like quadrature amplitude modulation (QAM) or NRZ are used, the received signal at any time depends on multiple transmitted symbols. In this case the complexity of equalization in serial schemes which use time domain equalization rises rapidly. In contrast, the complexity of OFDM, and of systems using serial modulation and frequency domain equalization, scale well as data rates and dispersion increase.

A second major advantage of OFDM is that it transfers the complexity of transmitters and receivers from the analog to the digital domain. For example, while the precise design of analog filters can have a major impact on the performance of serial modulation systems, in OFDM any phase variation with frequency can be corrected at little or no cost in the digital parts of the receiver. Despite these important advantages of OFDM, it is only recently that it has been considered for optical communications.

While many details of OFDM systems are very complex, the basic concept of OFDM is quite 
simple. Data is transmitted in parallel on a number of different frequencies, and as a result the symbol period is much longer than for a serial system with the same total data rate. Because the symbol period is longer, ISI affects at most one symbol, and equalization is simplified. In most OFDM implementations any residual ISI is removed by using a form of guard interval called a cyclic prefix.

Fig.2 shows the block diagram of OFDM system.

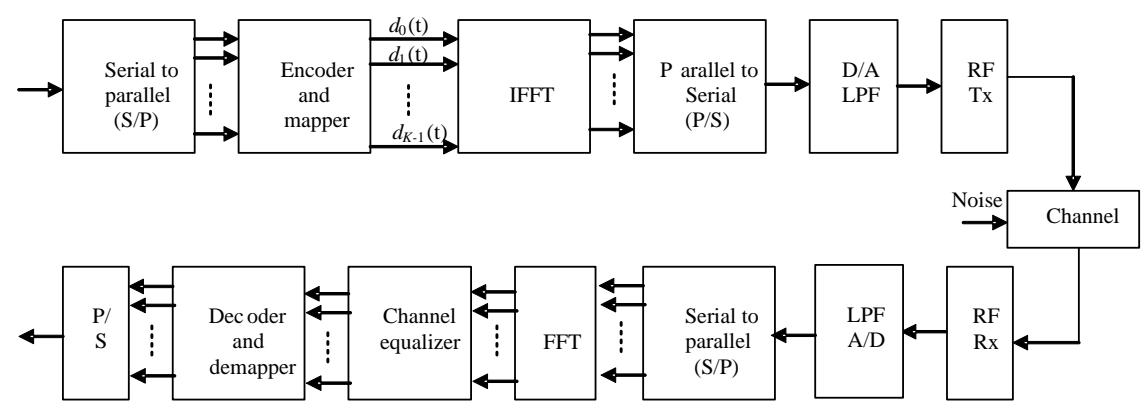

Fig.2: OFDM system model

The message bits are first converted into the parallel streams by OFDM transmitter which will be subsequently encoded and mapped into a sequence of symbols. Each symbol from encoder and mapper is carried out by the different sub carrier. Let $d_{l}(t)$ denotes the transmit symbol of the lth sub carrier at time $t, l=0,1,2 \cdots, L-1, L$ is the number of sub carriers. After IFFT, OFDM symbols are converted into the serial streams by $\mathrm{P} / \mathrm{S}$. These serial signals are processed through the $\mathrm{D} / \mathrm{A}$ converter, low passing filter (LPF), and upward frequency converter, and are sent to the channel. The demodulation process of OFDM signal in the receiver is the inverse process of its modulation.

OFDM signal $s(t)$ can be represented as

$$
s(t)=\sum_{l=0}^{L-1} d_{l}(t) \cdot e^{j \omega_{l} t}
$$

Where $\omega_{l}=\omega_{c}+l \Delta \omega$

$\omega_{c}$ is the frequency of the carrier.

Assume $d_{l}(t)$ is the fix value in one symbol period, such that

$$
d_{l}(t)=d(l)
$$

Assume the signal sampling frequency is $1 / T_{s}$, such that the signal sampling value can be represented as

$$
s\left(n T_{s}\right)=\sum_{l=0}^{L-1} d(l) e^{j\left(\omega_{c}+l \Delta \omega\right) n T_{s}}
$$

Assume one symbol period $T_{f}$ contains $K$ sampling values, such that

$$
T_{f}=K T_{s}
$$

Assume $\omega_{c}=0$, such that

$$
s\left(n T_{s}\right)=\sum_{l=0}^{L-1} d(l) e^{j(l \Delta \omega) n T_{s}}
$$

From the formula (10), it can be shown that the modulation and demodulation of OFDM system can be realized by the IFFT and FFT.

\section{MIMO-OFDM Model}

In wireless communications, spectrum is a scarce resource and hence imposes a high cost on the high data rate transmission. Fortunately, the emergence of multiple antenna system has opened 
another very resourceful dimension-space, for information transmission in the air. It has been demonstrated that multiple antenna system provides very promising gain in capacity without increasing the use of spectrum, reliability, throughput, power consumption and less sensitivity to fading, hence leading to a breakthrough in the data rate of wireless communication systems. Since then, MIMO system has become one of the major focuses in the research community of wireless communications and information theory. We use ultra wideband (UWB) OFDM along with MIMO system. OFDM is a multi-carrier system, the available spectrum is divided into many narrow bands and data is divided in parallel streams each transmitted on a separate band. OFDM gives a simple way of dealing with multi-path. The applications of OFDM include Digital Television, Asymmetric Digital subscriber loop (ADSL) and future mobile telephony.

The MIMO-OFDM technology, which is composed of MIMO technology and OFDM technology, is regarded as the core technology of B3G and 4G of wireless wideband communication and wireless network of next generation. From above analyzing, MIMO and OFDM techniques can resolve the problems of bandwidth efficiency and multi-path fading. MIMO technique can propagate multiplexing data streams through the individual parallel channels, and it will increase the spectrum efficiency without increasing the system bandwidth. OFDM technique can convert the frequency selective fading of the channel to the flat fading in the frequency domain, and decrease the multi-path fading influence[7]. The combined of these two techniques has two advantages mainly. One is very high data transmit rate. Another is every strong reliability through the diversity. It can be shown from the research that the OFDM system is combined with MIMO technique very suitably[8].

Fig.3 shows the block diagram of OFDM system.

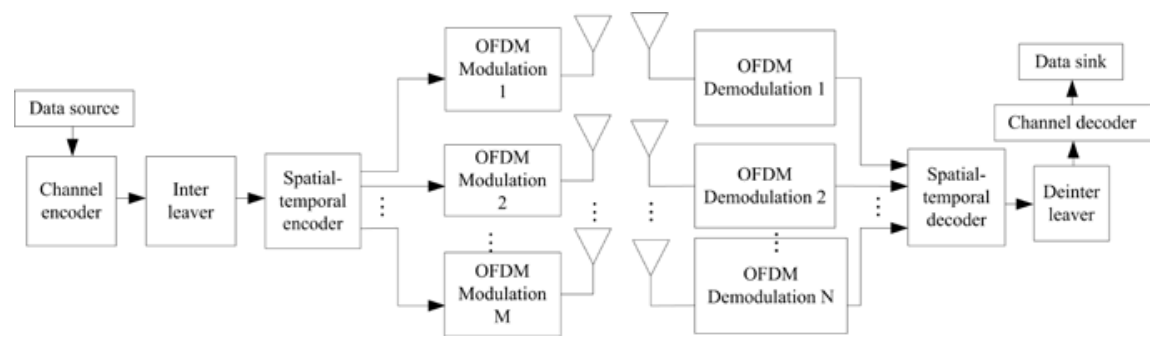

Fig.3: MIMO-OFDM system model

Assume that there are $M$ transmit antennas and $N$ receive antennas in system. It is defined that $h_{m, n}^{i, l}$ is the pulse response of the lth path from the $m$ th transmit antenna to the $n$th receive antenna. In $h_{m, n}^{i, l}, i$ represents the $i$ th frame of signal, and there are $N_{b}$ OFDM symbols. The channel pulse response of $h_{m, n}^{i, l}$ keeps unchanged in the time of each frame signal. $X_{m, k}^{i}(j)$ is the $j$ th symbol of the $i$ th frame of signal in the $k$ th sub-carrier from the $m$ th transmit antenna, and $Y_{n, k}^{i}(j)$ is the $j$ th symbol of the $i$ th frame of signal in the $k$ th sub-carrier received by the $n$th receive antenna. $V_{n, k}^{i}(j)$ is the adding white gauss noise of the $k$ th sub-carrier in the $n$th receive antenna, whose mean is zero, and variance is $\sigma_{v}^{2}$. The frequency domain response of the $k$ th sub-carrier of channel can be represented as

$$
H_{m, n}^{i, k}=\sum_{l=0}^{L-1} h_{m, n}^{i, l} W_{N_{c}}^{k l}, m=1, \ldots, M, n=1, \ldots, N, k=0, \ldots, N_{c}-1
$$

Where $N_{c}$ is the number of sub-carrier.

$L$ is the length of channel, and $L<N_{c}, N_{c}=g L$.

$W_{N_{c}}=e^{-\bar{j} 2 \pi / N_{c}}(\bar{j}=\sqrt{-1})$.

DFT matrix $\mathbf{F}_{\mathbf{N}_{\mathrm{c}}}$ of $N_{c} \times N_{c}$ dimensions is constructed. Its element $(k, l)$ is $W_{N_{c}}^{(k-1)(l-1)}$.

To analyze simply, the relation formula between the channel pulse response and the frequency domain response represented by the above formula (11) can be given as 


$$
\mathbf{H}^{\mathbf{i}}=\mathbf{F}_{N N_{c}}(:, 1: N L) \mathbf{h}^{i}
$$

Where $\mathbf{H}^{i}=\left[\left(\mathbf{H}^{i, 0}\right)^{\mathrm{T}}, \cdots,\left(\mathbf{H}^{i,\left(N_{c}-1\right)}\right)^{\mathrm{T}}\right]^{\mathrm{T}}$.

$\mathbf{F}_{N N_{c}}=\mathbf{F}_{N_{c}} \otimes \mathbf{I}_{N}, \otimes$ is the Kronecker product operation.

$\mathbf{F}_{N N_{c}}(:, 1: N L)$ is the first $N L$ rows of matrix $\mathbf{F}_{N N_{c}}$.

$\mathbf{H}^{i, k}=\left[\begin{array}{ccc}H_{1,1}^{i, k} & \cdots & H_{M 1}^{i, k} \\ \vdots & \ddots & \vdots \\ H_{1, N}^{i, k} & \cdots & H_{M, N}^{i, k}\end{array}\right]$.

$\mathbf{h}^{i}=\left[\left(\mathbf{h}^{i, 0}\right)^{T}, \cdots,\left(\mathbf{h}^{i,(L-1)}\right)^{T}\right]^{T}$.

$\mathbf{h}^{i, l}=\left[\begin{array}{ccc}h_{1,1}^{i, l} & \cdots & h_{M, 1}^{i, l} \\ \vdots & \ddots & \vdots \\ h_{1, N}^{i, l} & \cdots & h_{M, N}^{i, l}\end{array}\right]$.

According to the frequency domain response of the sub-carrier of channel, the demodulated signal in $n$th receive antenna can be given as

$$
Y_{n, k}^{i}(j)=\sum_{m=1}^{M} H_{m, n}^{i, k} X_{m, k}^{i}(j)+V_{n, k}^{i}(j), \quad n=1, \ldots, N, k=0, \ldots, N_{c}-1
$$

The received signals of $N$ receive antennas can be given as

Where $\mathbf{Y}_{k}^{i}(j)=\left[Y_{1, k}^{i}(j), \cdots, Y_{N, k}^{i}(j)\right]^{\mathrm{T}}$.

$$
\mathbf{Y}_{k}^{i}(j)=\mathbf{H}^{i, k} \mathbf{X}_{k}^{i}(j)+\mathbf{V}_{k}^{i}(j), \quad k=0 \ldots N_{c}-1
$$

$\mathbf{X}_{k}^{i}(j)=\left[X_{1, k}^{i}(j), \cdots, X_{M, k}^{i}(j)\right]^{\mathrm{T}}$.

$\mathbf{V}_{k}^{i}(j)=\left[V_{1, k}^{i}(j), \cdots, V_{N, k}^{i}(j)\right]^{\mathrm{T}}$.

The received signals can be represented as the following matrix

$$
\mathbf{Y}^{i}(j)=\mathbf{X}_{1}^{i}(j) \mathbf{H}^{i}(:, 1)+\cdots+\mathbf{X}_{M}^{i}(j) \mathbf{H}^{i}(:, M)+\mathbf{V}^{i}(j)
$$

Where $\mathbf{Y}^{i}(j)=\left[\left(\mathbf{Y}_{0}^{i}(j)\right)^{\mathrm{T}}, \cdots,\left(\mathbf{Y}_{N_{c}-1}^{i}(j)\right)^{\mathrm{T}}\right]^{\mathrm{T}}, \quad \mathbf{X}_{m}^{i}(j)=\left\{\operatorname{diag}\left[X_{m, 0}^{i}(j), \cdots, X_{m, N_{c}}^{i}(j)\right]^{\mathrm{T}}\right\} \otimes \mathbf{I}_{N}$, $\mathbf{V}^{i}(j)=\left[\left(\mathbf{V}_{0}^{i}(j)\right)^{\mathrm{T}}, \cdots,\left(\mathbf{V}_{N_{c}-1}^{i}(j)\right)^{\mathrm{T}}\right]^{\mathrm{T}}, \mathbf{H}^{i}(:, q)=\left[\left(\mathbf{H}^{i, 0}(:, q)\right)^{\mathrm{T}},\left(\mathbf{H}^{\mathrm{i}, 1}(:, q)\right)^{\mathrm{T}}, \cdots,\left(\mathbf{H}^{i,\left(N_{c}-1\right)}(:, q)\right)^{\mathrm{T}}\right]$ is $q$ th row of matrix $\mathbf{H}^{i}$, and $\mathbf{H}^{i, k}(:, q)$ is the $q$ th row of matrix $\mathbf{H}^{i, k}$.

\section{Conclusions}

The combined technique of MIMO-OFDM has been regarded as the core technique of new generation wireless communication and next generation wireless LAN. It adopts the antenna array to realize the spatial diversity in OFDM system, and increases the received signal quality. MIMOOFDM technique adopts the temporal, frequency, and spatial diversity techniques in the mean while, and increases the capability of resisting noise, interference, and multi-path greatly.

\section{Acknowledgements}

This work was financially supported by My colleague Aviation University of Air Force UAV Department of the professor Tao Jianwu's technical support in the academic and experimental testing.

\section{References}

[1] Liu Hui, Zhang Fu-Chun, Huang Guo-hua. A new receiving algorithm in MIMO-OFDM[J]. CHINESE JOURNAL OF AERONAUTICS, 30 (3) , pp. 484-489,2009.

[2] H Bolcskei, D Gesbert, A J Paulraj. On the capacity of OFDM-based spatial multiplexing systems[J], IEEE Transactions on Communications, 50(2) ,pp. 225-234,2002. 
[3] V Tarokh, H Jafarkahani, A R Calderbank. Space-time block codes for wireless communications: performance results[J], IEEE Journal on Selected Areas in Communications, 17(3) , pp. 451460,1999.

[4] G J Foschini, M J Gans. On limits of wireless communications in a fading environment when using multiple antennas[J],Wireless Personal Communications, 6(3) , pp.311 335, 1998.

[5] G J Foschini, G D Golden, R A Valenzuela. Simplified processing for high spectral efficiency wireless communication employing multi-element arrays[J], IEEE Journal on Selected Areas in Communications,17(11), pp.1841 1852, 1999.

[6] R van Nee, R Prasad. OFDM for Wireless Multimedia Communications[M], Artech House, 2000.

[7] Heidi, Steendam, Moeneclaey. Analysis and optimization of the performance of OFDM on frequency-selective time-selective fading channels[J], IEEE transactions on communication, 47(12): pp.1811-1819,1999.

[8] Agrawal D, Tarokh V, Nagu ib A. Space-time coded OFDM for high data rate wireless communication over wideband channels[J], Proc. Of Vehicular Technology Conf. VTC'98. Ottawa, Ont., Canada, 5: pp.2232-2236 ,1998. 